\title{
A Probabilistic Approach to Spectral Unmixing
}

\author{
Cong Phuoc Huynh ${ }^{1}$ and Antonio Robles-Kelly ${ }^{1,2}$ \\ 1 School of Engineering, Australian National University, Canberra ACT 0200, Australia \\ 2 National ICT Australia (NICTA) ${ }^{\star}$, Locked Bag 8001, Canberra ACT 2601, Australia
}

\begin{abstract}
In this paper, we present a statistical approach to spectral unmixing with unknown endmember spectra and unknown illuminant power spectrum. The method presented here is quite general in nature, being applicable to settings in which sub-pixel information is required. The method is formulated as a simultaneous process of illuminant power spectrum prediction and basis material reflectance decomposition via a statistical approach based upon deterministic annealing and the maximum entropy principle. As a result, the method presented here is related to soft clustering tasks with a strategy for avoiding local minima. Furthermore, the final endmembers depend on the similarity between pixel reflectance spectra. Hence, the method does not require a preset number of material clusters or spectral signatures as input. We show the utility of our method on trichromatic and hyperspectral imagery and compare our results to those yielded by alternatives elsewhere in the literature.
\end{abstract}

\section{Introduction}

Spectral unmixing is commonly stated as the problem of decomposing an input spectral signal into relative portions of known spectra of endmembers. The endmembers can be any man-made or naturally occurring materials such as water, metals, etc. The input data varies in many forms, such as radiance or reflectance spectra, or hyperspectral images. The problem of unmixing applies to all those cases where a capability to provide subpixel detail is needed, such as geosciences, food quality assessment and process control. Moreover, unmixing can be viewed as a pattern recognition task related to soft-clustering with known or unknown endmembers.

Current unmixing methods assume availability of the endmember spectra [1]. This yields a setting in which cumbersome labelling of the endmember data is effected through expert intervention. Added to the complexity of endmember labeling is the fact that, often, illumination is a confounding factor in determining the intrinsic surface material reflectance. As a result, in general, unmixing can be viewed as a dual challenge. Firstly, endmembers shall, in case of necessity, be identified automatically. Secondly, reflectance has to be recovered devoid of illumination and scene geometry. The former of these can be viewed as an instance of blind-source or unsupervised clustering techniques. The latter is a photometric invariance problem.

\footnotetext{
* NICTA is funded by the Australian Government as represented by the Department of Broadband, Communications and the Digital Economy and the Australian Research Council through the ICT Centre of Excellence program.
} 
Spectral unmixing with automatic endmember extraction is closely related to the simultaneous estimation of illuminant power spectrum and material reflectance. Many of the methods elsewhere in the literature hinge on the notion that the simultaneous recovery of the reflectance and illuminant power spectrum requires an inference process driven by statistical techniques. In [2], Stainvas and Lowe proposed a Markov Random Field to separate illumination from reflectance from the input images. On the other hand, the physics-based approach in [3] for image colour understanding alternately forms hypotheses of colour clusters from local image data and verifies whether these hypotheses fit the input image. More recently, in [4], Tajima performed an imperfect segmentation of the object colours in images by recursively subdividing the scene's color space through the use of Principal Component Analysis. Li et al. [5] proposed an energy minimisation approach to estimating both the illumination and reflectance from images.

Note that the methods above aim at tackling either the colour constancy or the image segmentation problem and do not intend to recover subpixel information. Here, we present an approach to spectral unmixing with unknown lighting conditions and unknown endmember signatures. Unlike previous approaches related to the field of spectral unmixing [6] and photometric invariants [7], our method does not assume known power spectrum or colour of the illuminant. We adopt a probabilistic treatment of the problem which allows for a soft clustering operation on the pixel reflectance spectra. Thus, the method is quite general in the sense that it is applicable to any number of colour channels assuming no prior knowledge of the illumination condition as well as the surface reflectance.

The paper is organised as follows. In section 2 we cast the problem of simultaneous spectral unmixing and illumination recovery in a probabilistic framework based on the dichromatic reflection model [8] and the maximum entropy principle [9]. In this section, we also describe a deterministic annealing approach to solve the problem for a single spectral radiance image with unknown lighting condition and endmembers. Section 3 illustrates the utility of our method on real-world multispectral and trichromatic images.

\section{Probabilistic Formulation}

This section provides a probabilistic formulation of the problem of spectral unmixing on multispectral imagery. To commence, we pose the problem in the general case as a minimisation one governed by the interaction between image pixels and endmembers. This yields a general formulation for spectral radiance images with no prior knowledge of the lighting condition. By making use of the dichromatic model [8] and the maximum entropy principle [9], our integrated spectral unmixing and illumination estimation algorithm involves three interleaved steps

1. From the input spectral radiance image, find an optimal set of dichromatic hyperplanes representing the current endmembers and material association probabilities per pixel.

2. Estimate the illumination power spectrum making use of the least-squares intersection between the dichromatic hyperplanes.

3. Recover the endmembers from the reflectance image. The reflectance image is obtained via the normalisation of the input radiance image with respect to the current estimate of the illumination power. 


\subsection{General Unmixing Formulation}

Our general problem is formulated as follows. Given an input multispectral image $\mathcal{I}$, we aim to recover the basis material reflectance, i.e. endmember signatures, as well as their relative proportions at each pixel in a single trichromatic or multi-band image. Let $\mathcal{M}$ be the set of unknown endmembers in the scene under study. Here we take a probabilistic viewpoint on the problem by equating material composition to the notion of association probability relating the input signal at a pixel $u$ to a basis material $M \in \mathcal{M}$, which we denote as $p(M \mid u)$. The problem also involves a definition of the affinity between the input signal at a pixel $u$ and a basis material $M$, which we denote as $d(u, M)$. The spectral unmixing statement is then cast as minimising the expected affinity between the given image $\mathcal{I}$ and the endmembers.

Thus, we aim to find a distribution of material association probabilities $\mathcal{P}=$ $\{p(M \mid u) \mid M \in \mathcal{M}, u \in \mathcal{I}\}$ that minimises the total expected pixel-material affinity

$$
C_{\text {Total }}=\sum_{u \in \mathcal{I}} \sum_{M \in \mathcal{M}} p(M \mid u) d(u, M)
$$

subject to the law of total probability $\sum_{M \in \mathcal{M}} p(M \mid u)=1 \forall u \in \mathcal{I}$.

Note that the formulation in Equation[1] is reminiscent of a soft clustering problem. Here we aim to find the optimal association of image pixels with a set of materials which minimises the cost function. Since the above formulation often favours single-material composition per pixel as each pixel is finally associated with its closest material with probability one, we restate the problem as that of finding a distribution of material association $\mathcal{P}$ that minimises the above cost function subject to the maximum entropy criterion [10]. The justification of the additional constraint originates from the maximum entropy principle [9], which states that amongst all the probability distributions that satisfy a set of constraints, the one with the maximum entropy is preferred.

To apply this principle to our problem, let us fix the expected affinity level at hand. While several distributions of material association satisfy this level of expected affinity, choosing non-maximal entropy distributions would imply making rather restrictive assumptions on the problem. As a result, only the one with the maximum entropy shall require no further constraints.

By making use of the entropy

$$
H(\mathcal{P})=-\sum_{u \in \mathcal{I}} \sum_{M \in \mathcal{M}} p(M \mid u) \log p(M \mid u)
$$

to quantify the level of uncertainty of the material association distribution we reformulate the expected material affinity as

$$
C_{\text {Entropy }}=C_{\text {Total }}-\mathcal{L}
$$

where

$$
\mathcal{L}=T H(\mathcal{P})+\sum_{u \in \mathcal{I}} \alpha(u)\left(\sum_{M \in \mathcal{M}} p(M \mid u)-1\right)
$$

in which $T \geq 0$ and $\alpha(u)$ are Lagrange multipliers. Note that $T \geq 0$ weighs the level of randomness of the material association probabilities whereas $\alpha(u)$ enforces the total probability constraint for every image pixel $u$. 


\subsection{Illumination Spectrum Estimation}

With the expression in Equation 3 at hand, we now proceed to integrate the dichromatic reflection theory introduced by Shafer $[8]$ into the general problem formulation in Section 2.1

The dichromatic model for a scene illuminated by a single illumination source relates the observed image radiance to the illuminant power spectrum and material reflectance. According to the dichromatic model, the observed colour or spectral radiance power at a point in the scene is a linear combination of the body reflection and interface reflection. The former component is affected by the material reflectance while the latter one is purely governed by the illuminant power spectrum. Therefore, the spectral radiance spectrum at a surface point composed of a single material belongs to a two-dimensional linear subspace spanned by the illuminant spectrum and the diffuse radiance spectrum of the material. We refer to this subspace as the dichromatic hyperplane.

In the most general case where each scene location is made of a mixture of materials, the pixel radiance spectrum does not necessarily lie in any of the dichromatic hyperplanes corresponding to the endmembers. Therefore, it is natural to quantify the notion of pixel-material affinity as the distances between the pixel radiance spectrum and the dichromatic planes corresponding to the endmembers. A zero-distance means purity in terms of material composition. The further the distance, the lower the proportion of the basis material or endmember.

To define the spectral unmixing problem using the dichromatic reflection model we require some formalism. Let us consider a multispectral imaging sensor that samples the spectral dimension of incoming light at wavelengths $\lambda_{1}, \ldots \lambda_{K}$. The input radiance at pixel $u$ and wavelength $\lambda_{i}$ is denoted as $I\left(u, \lambda_{i}\right)$ and the spectral component of the illumination power at wavelength $\lambda_{i}$ is $L\left(\lambda_{i}\right)$. For brevity, we adopt thes vectorial notations of the illumination spectrum, the input radiance spectrum at each pixel $u$ and the material reflectance spectrum of material $M$, which we denote $\mathbf{L}, \mathbf{I}(u)$ and $\mathbf{S}(M)$, respectively.

As discussed above, we characterise the combination of illumination power spectrum and material basis in a scene as a set of basis dichromatic planes, each of which captures all the possible radiance spectra reflected from a point made of a single basis material. Each of these two-dimensional planes can be further specified by two basis vectors. Note that the choice of basis vectors is, in general, arbitrary. Let us denote the dichromatic hyperplane for the endmember material $M$ as $\mathcal{Q}(M)$, with two basis column-vectors $\mathbf{z}_{1}(M), \mathbf{z}_{2}(M)$.

With these ingredients, the affinity between the pixel $u$ and the basis material $M$ is quantified as the orthogonal distance between a $K$-dimensional point representing its radiance spectrum $\mathbf{I}(u)$ and the hyperplane $\mathcal{Q}(M)$. Since the linear projection matrix onto $\mathcal{Q}(M)$ is defined as $Q(M)=A(M)\left(A(M)^{T} A(M)\right)^{-1} A(M)^{T}$, where $A(M)=$ $\left[\mathbf{z}_{1}(M), \mathbf{z}_{2}(M)\right]$, the affinity distance is therefore defined as the squared $L^{2}$-norm of the hyperplane $d(u, M)=\|\mathbf{I}(u)-Q(M) \mathbf{I}(u)\|^{2}$

The unmixing problem on spectral radiance images becomes that of seeking for an optimal set of linear projection matrices $\{Q(M), M \in \mathcal{M}\}$ corresponding to the endmembers and the material association probabilities $p(M \mid u)$ that minimise the following function 


$$
C_{\text {Light }}=\sum_{u \in \mathcal{I}} \sum_{M \in \mathcal{M}} p(M \mid u)\|\mathbf{I}(u)-Q(M) \mathbf{I}(u)\|^{2}-\mathcal{L}
$$

For the recovery of the optimal dichromatic hyperplanes, we fix the material association probability distribution. In this situation, we recast the problem as that of finding the optimal basis vectors $\mathbf{z}_{1}(M), \mathbf{z}_{2}(M)$ for each endmember $M$ so as to minimise the expected material affinity given by the first term on the right-hand side of Equation 5

$$
\sum_{u \in \mathcal{I}} p(M \mid u)\|\mathbf{I}(u)-Q(M) \mathbf{I}(u)\|^{2}=\sum_{u \in \mathcal{I}}\|\sqrt{p(M \mid u)} \mathbf{I}(u)-A(M) b(u, M)\|^{2}
$$

where $A(M)=\left[\mathbf{z}_{1}(M), \mathbf{z}_{2}(M)\right]$ and $b(u, M) \triangleq \sqrt{p(M \mid u)}\left(A(M)^{T} A(M)\right)^{-1} A(M)^{T} \mathbf{I}(u)$ is a two-element column vector.

We note that the right-hand side of Equation 6 is the Frobenius norm of the matrix $\mathbb{I}-\mathbb{J}$, where $u_{1}, u_{2}, \ldots u_{N}$ are all the image pixels and

$$
\begin{aligned}
\mathbb{I} & =\left[\sqrt{p\left(M \mid u_{1}\right)} \mathbf{I}\left(u_{1}\right), \sqrt{p\left(M \mid u_{2}\right)} \mathbf{I}\left(u_{2}\right), \ldots, \sqrt{p\left(M \mid u_{N}\right)} \mathbf{I}\left(u_{N}\right)\right] \\
\mathbb{J} & =A(M)\left[b\left(u_{1}, M\right) b\left(u_{2}, M\right), \ldots, b\left(u_{N}, M\right)\right]
\end{aligned}
$$

Since $\operatorname{rank}(\mathbb{J}) \leq \operatorname{rank}(A(M))=2$, the problem above amounts to finding a matrix $\mathbb{J}$ with rank at most 2 that best approximates the known matrix $\mathbb{I}$. We achieve this via a Singular Value Decomposition operation such that $\mathbb{I}=U \Sigma V$, where $\mathrm{U}$ and $\mathrm{V}$ are the left and right singular matrices of $\mathbb{I}$ and $\Sigma$ is a diagonal matrix containing its singular values. The solution to this problem is then given by $\mathbb{J}=U \Sigma^{*} V$, in which the only non-zero singular values of $\Sigma^{*}$ are the two leading singular values of $\Sigma$. The vectors $\mathbf{z}_{1}(M), \mathbf{z}_{2}(M)$ correspond to the two leading eigenvectors of $\mathbb{I}$, i.e. those corresponding to the non-zero singular values in $\Sigma^{*}$. With the basis vectors $\mathbf{z}_{1}(M), \mathbf{z}_{2}(M)$ at hand, we can estimate the illumination power spectrum as a least-squares intersection between dichromatic hyperplanes making use of the algorithm in [11].

\subsection{Endmembers from Image Reflectance}

With the illuminant power spectrum at hand, we can obtain the reflectance image from the input radiance image by illumination normalisation. Let the reflectance spectrum at each image pixel $u$ be a wavelength-indexed vector $\mathbf{R}(u)=\left[R\left(u, \lambda_{1}\right), \ldots, R\left(u, \lambda_{K}\right)\right]^{T}$, where $R\left(u, \lambda_{1}\right)$ is given by $R(u, \lambda)=\frac{I(u, \lambda)}{L(\lambda)}$. Note that the affinity distance between a pixel reflectance spectrum and a material reflectance spectrum can be defined based on their Euclidean angle. Mathematically, the distance is given as follows

$$
d(u, M)=1-\frac{\tilde{\mathbf{R}}(u)^{T} \mathbf{S}(M)}{\|\mathbf{S}(M)\|}
$$

where $\tilde{\mathbf{R}}(u)$ has been obtained by normalising $\mathbf{R}(u)$ to unit $L^{2}$-norm.

With this affinity distance, our unmixing problem becomes that to find a set of basis material reflectance spectra and a distribution of material association probabilities 
$p(M \mid u)$ for each pixel $u$ and material $M$ that minimise the following cost function

$$
C_{\text {Reflectance }}=\sum_{u \in \mathcal{I}} \sum_{M \in \mathcal{M}} p(M \mid u)\left(1-\frac{\tilde{\mathbf{R}}(u)^{T} \mathbf{S}(M)}{\|\mathbf{S}(M)\|}\right)-\mathcal{L}
$$

We now derive the optimal set of endmember spectra so as to minimise the cost function $C_{\text {Reflectance }}$ in Equation 8 . To minimise the cost function, we compute the derivatives of $C_{\text {Reflectance }}$ with respect to the endmember reflectance $\mathbf{S}(M)$ to yield

$$
\frac{\partial C_{\text {Reflectance }}}{\partial \mathbf{S}(M)}=-\sum_{u \in \mathcal{I}} p(M \mid u) \frac{\|\mathbf{S}(M)\|^{2} \tilde{\mathbf{R}}(u)-\left(\tilde{\mathbf{R}}(u)^{T} \mathbf{S}(M)\right) \mathbf{S}(M)}{\|\mathbf{S}(M)\|^{3}}
$$

Setting this derivative to zero, we obtain

$$
\mathbf{S}(M) \propto \sum_{u \in \mathcal{I}} p(M \mid u) \tilde{\mathbf{R}}(u)
$$

\subsection{Material Association Probability Recovery}

Note that, in the cost functions associated to the steps above, we require the material association probability $p(M \mid u)$ to be at hand. In this section, we describe how $p(M \mid u)$ can be recovered via deterministic annealing. A major advantage of the deterministic annealing approach is that it mitigates attraction to local minima. In addition, deterministic annealing converges faster than stochastic or simulated annealing [12].

The deterministic annealing approach casts the Lagrangian multiplier $T$ in the role of the system temperature in an analogous annealing process used in statistical physics. Initially, the whole process starts at a high temperature. At each temperature, the system eventually converges to a thermal equilibrium. After reaching this state, the system experiences a "phase transition" as the temperature is lowered. The optimal parameters corresponding to the equilibrium state are tracked through such phase transitions. At zero temperature, we can directly minimise the expected pixel-material affinity $C_{\text {Entropy }}$ to obtain the final material association probabilities $\mathcal{P}$ and the endmember reflectance.

The recovery of the endmembers in step 3 described in Section 2 is, hence, somewhat similar to a soft-clustering process. At the beginning, this process is initialised at a high temperature with a single endmember by assuming all the image pixels are made of the same material. As the temperature is lowered, the set of endmembers grows. This, in essence, constitutes several "phase transitions", at which new endmembers arise from the existing ones. This phenomenon is due to the discrepancy in the affinity between the image pixels and the existing endmembers.

At each phase of the annealing process, where the temperature $T$ is kept constant, the algorithm proceeds as two interleaved minimisation steps at each iteration so as to arrive at an equilibrium state. These two minimisation steps are performed alternately with respect to the material association probabilities $\mathcal{P}$ and the endmembers as captured by the pixel-material affinity function $d(u, M)$. For the recovery of the material association probabilities, we fix the endmember set and seek for the probability distribution which 
minimises the cost function $C_{\text {Entropy }}$ in Equation 3 . This is achieved by setting the partial derivative $\frac{\partial C_{E n t r o p y}}{\partial p(M \mid u)}=d(u, M)+T \log p(M \mid u)+T-\alpha(u)$ to zero. We obtain

$$
p(M \mid u)=\exp \left(\frac{-d(u, M)}{T}+\frac{\alpha(u)}{T}-1\right) \propto \exp \left(\frac{-d(u, M)}{T}\right) \forall M, u
$$

Since $\sum_{M \in \mathcal{M}} p(M \mid u)=1$, it can be shown that the optimal material association probability distribution for a fixed endmember set $\mathcal{M}$ is given by the Gibbs distribution

$$
p(M \mid u)=\frac{\exp \left(\frac{-d(u, M)}{T}\right)}{\sum_{M^{\prime} \in \mathcal{M}} \exp \left(\frac{-d\left(u, M^{\prime}\right)}{T}\right)}
$$

\section{Experiments}

In this section, we provide validation results of our algorithm on real-world multispectral and trichromatic imagery. To perform the validation task, we used two datasets. The first of these comprises 321 Mondrian and specular images from the Simon Fraser University database [13]. In addition, we have acquired an image database of 51 human subjects, each captured under one of 10 light sources with varying directions and spectral power. The multispectral imagery has been acquired using a pair of benchtop hyperspectral cameras. Each of which is equipped with Liquid Crystal Tunable Filters which are capable of resolving up to $10 \mathrm{~nm}$ in both the visible $(430-720 \mathrm{~nm})$ and near infrared $(650-990 \mathrm{~nm})$ wavelength ranges. The ground truth illuminant spectra have been measured using a white reference target, i.e. a Labsphere Spectralon.

On both the multispectral and trichromatic image databases, we configure the deterministic annealing process with the initial and terminal temperatures of $T_{\max }=0.02$ and $T_{\min }=0.00025$. We employ an exponential decay function as the cooling schedule with a decay rate of 0.8 . The maximum number of endmembers in each image is set to 20 .

First, we provide results on the illuminant recovered by our algorithm. Since our method is an unmixing one which delivers at output the illuminant power spectrum and endmember reflectance, the error on the recovered illuminant is an indirect measure of the efficacy of the algorithm. Here we present a quantitative comparison with Colour Constancy methods that can be applied to single images with no pre-processing steps and no prior statistics of image colours gathered from a large number of images. These alternatives include the Grey-World [14] and White-Patch hypotheses [15] which are special instances of the Shades of Grey method [16], and the Grey-Edge method [17]. The accuracy of the estimated multispectral illumination power and trichromatic illuminant colour is quantified as the Euclidean angle with respect to the ground truth.

In the fourth and fifth columns of Table 1, we present the trichromatic illuminant estimation results on the Simon Fraser University dataset [13]. These include the Mondrian and specular datasets with 8 and 16-bit dynamic ranges. Overall our method yields better results than the Grey-World method and is quite comparable to the White-Patch method. The other methods outperform ours but the difference in performance is less than two degrees. Recall that the illumination estimation method we employ is purely 
Table 1. A comparison of the illumination recovery performance of our method with a number of alternatives. The angular error (in degrees) are shown for both multispectral and trichromatic image datasets.

\begin{tabular}{|c||c|c||c|c|}
\hline \multicolumn{1}{|c||}{ Method } & \multicolumn{2}{c|}{ Multispectral Images } & \multicolumn{2}{c|}{ Trichromatic Images } \\
\cline { 2 - 5 } & Visible Range & Infrared Range & 8-bit & 16-bit \\
\hline Our method & $6.84 \pm 3.92$ & $4.19 \pm 4.75$ & $8.24 \pm 8.73$ & $7.99 \pm 7.59$ \\
\hline Grey-World [14] & $8.44 \pm 3.03$ & $6.89 \pm 2.23$ & $9.75 \pm 9.4$ & $9.67 \pm 9.25$ \\
\hline White-Patch [15] & $11.91 \pm 8.02$ & $8.53 \pm 6.22$ & $7.66 \pm 6.92$ & $7.70 \pm 6.92$ \\
\hline Shades of Grey (sixth-order norm) [16] & $8.17 \pm 4.61$ & $5.16 \pm 3.65$ & $6.23 \pm 6.50$ & $6.27 \pm 6.47$ \\
\hline Grey-Edge (first-order norm) [17] & $6.23 \pm 2.06$ & $2.21 \pm 1.07$ & $6.78 \pm 4.37$ & $6.82 \pm 4.35$ \\
\hline Grey-Edge (sixth-order norm) [17] & $8.37 \pm 7.77$ & $6.45 \pm 5.86$ & $6.42 \pm 5.82$ & $6.45 \pm 5.82$ \\
\hline
\end{tabular}

based on dichromatic patches [11], i.e. its stability and robustness improve with an increasing number of materials and a higher level of image specularity. Therefore, it is not surprising that the illumination estimates are severely affected by the large number of images in this trichromatic database that are either completely diffuse or consist of only a few materials.

In the second and third columns we show the accuracy of the illumination spectra recovered from the multispectral images. On the multispectral images, our method clearly outperforms all instances of the Shades of Grey method and the Grey-Edge method implemented with a sixth-order Minkowski norm, by a significant margin. Note that the Shades-of-Grey paradigm relies on the heuristics that the average scene colour is achromatic. Therefore the accuracy of these methods is somewhat limited by the degree of achromaticity of the average colours in the multispectral images. The Grey-Edge method with a first-order Minkowski norm performs better than our method mainly because of the abundance of edges and material boundaries in the multispectral images. However, it should be stressed that the former method does not recover endmembers and material composition.

Now we turn our attention to the performance of our algorithm for the spectral unmixing task on the multispectral image database. We quantify the accuracy of the endmember reflectance extracted by our method from the multispectral images as compared to the ground truth measurements. To acquire the ground-truth endmembers, we normalise each input radiance image by its ground-truth illumination spectrum and then apply a K-means algorithm on the reflectance image to produce 20 clusters of pixels, each made of the same material. The resulting cluster centroids are deemed to be the ground-truth endmember reflectance. As before, we have computed the Euclidean angles between the basis material reflectance recovered by our method and those recovered by K-means clustering. The mean angular differences are 8.56 degrees for the visible and 11.49 degrees for the infrared spectrum, which are comparable to those produced by the alternative Colour Constancy methods shown before.

Next, we compare the association probability maps recovered by our method and the Spectral Angle Mapper (SAM) [18]. As an input to this operation the SAM, the illumination spectra are assumed to be those recovered by the Grey-Edge method with the first-order Minkowski norm. Recall that this method is the only Colour Constancy 

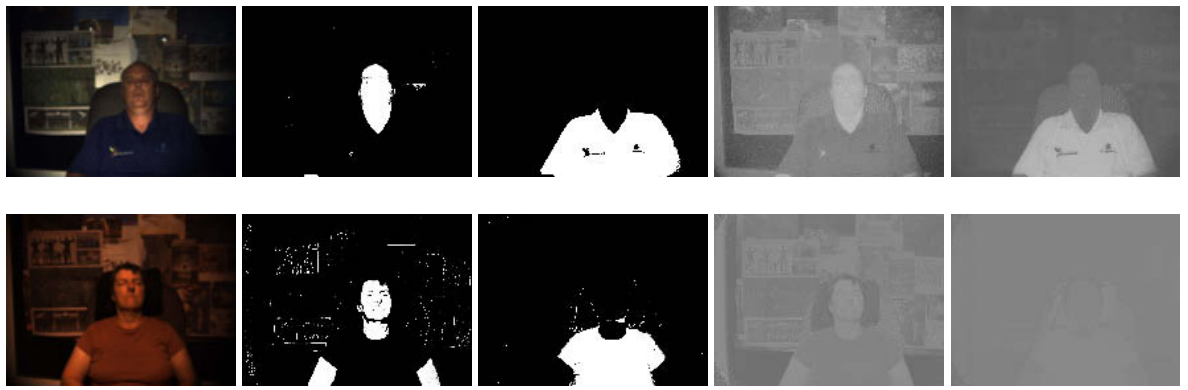

Fig. 1. Material maps estimated from a visible image (top row) and a near infrared image (bottom row). First column: the input image in pseudo colour. Second and third columns: the probability maps of skin and cloth produced by our method. Fourth and fifth columns: the probability maps of skin and cloth produced by the Spectral Angle Mapper(SAM).

method shown before that slightly outperforms our method in multispectral illumination spectrum recovery. For the SAM, the endmember reflectance spectra are those resulting from K-means clustering on the multispectral images.

In the second and third columns of Figure 1, we show the probability maps of skin and cloth materials recovered by our method. In the fourth and fifth columns, we show the probability maps recovered by the SAM. The two sample images shown have been captured under different illumination conditions, one of which in the visible and the other in the infrared spectrum. In the panels, the brightness of the probability maps is proportional to the association probability with the reference material. It is evident that our algorithm produces cleaner endmember maps than the SAM for both materials and spectral regions. In other words, our method correctly labels the skin and cloth regions as primarily composed of the respective ground truth endmember. We can attribute this to the ability of deterministic annealing in escaping from local minima. On the other hand, the SAM appears to assign a high proportion of non-primary materials to skin and cloth regions. In fact, the material maps in the fourth and fifth columns show a very weak distinction between the primary materials and the others in these regions. This symptom is not surprising since the SAM may not be able to determine the primary material in the case where a number of endmembers have nearly equal distances to the pixel reflectance spectrum.

\section{Conclusions}

We have introduced a probabilistic method for simultaneous spectral unmixing and illumination estimation provided no prior assumption on the lighting condition and the end-member spectra. We have formulated the unmixing task making use of the dichromatic reflection model [8] and the maximum entropy principle [9]. Moreover, we have used deterministic annealing as an optimisation method to improve convergence to the globally optimal solution. We have illustrated the utility of the method on hyperspectral and trichromatic imagery and compared our results against a number of alternatives. 


\section{References}

1. Lennon, M., Mercier, G., Mouchot, M.C., Hubert-moy, L.: Spectral unmixing of hyperspectral images with the independent component analysis and wavelet packets. In: Proc. of the International Geoscience and Remote Sensing Symposium (2001)

2. Stainvas, I., Lowe, D.: A generative model for separating illumination and reflectance from images. Journal of Machine Learning Research 4(7-8), 1499-1519 (2004)

3. Klinker, G.J., Shafer, S.A., Kanade, T.: A physical approach to color image understanding. Int. J. Comput. Vision 4(1), 7-38 (1990)

4. Tajima, J.: Illumination chromaticity estimation based on dichromatic reflection model and imperfect segmentation. In: Trémeau, A., Schettini, R., Tominaga, S. (eds.) CCIW 2009. LNCS, vol. 5646, pp. 51-61. Springer, Heidelberg (2009)

5. Li, C., Li, F., Kao, C., Xu, C.: Image Segmentation with Simultaneous Illumination and Reflectance Estimation: An Energy Minimization Approach. In: ICCV 2009: Proceedings of the Twelfth IEEE International Conference on Computer Vision (2009)

6. Bergman, M.: Some unmixing problems and algorithms in spectroscopy and hyperspectral imaging. In: Proc. of the 35th Applied Imagery and Pattern Recognition Workshop (2006)

7. Fu, Z., Tan, R., Caelli, T.: Specular free spectral imaging using orthogonal subspace projection. In: Proc. Intl. Conf. Pattern Recognition, vol. 1, pp. 812-815 (2006)

8. Shafer, S.A.: Using color to separate reflection components. Color Research and Applications 10(4), 210-218 (1985)

9. Jaynes, E.: On the rationale of maximum-entropy methods. In: Proceedings of the IEEE, 70(9), 939-952 (1982)

10. Jaynes, E.T.: Information Theory and Statistical Mechanics. Phys. Rev. 106(4), 620-630 (1957)

11. Finlayson, G.D., Schaefer, G.: Convex and Non-convex Illuminant Constraints for Dichromatic Colour Constancy. In: CVPR, vol. 1, pp. 598-604 (2001)

12. Kirkpatrick, S., Gelatt Jr., C.D., Vecchi, M.P.: Optimization by simulated annealing. Science 220, 671-680 (1983)

13. Barnard, K., Martin, L., Coath, A., Funt, B.V.: A comparison of computational color constancy Algorithms - Part II: Experiments with image data. IEEE Transactions on Image Processing 11(9), 985-996 (2002)

14. Buchsbaum, G.: A Spatial Processor Model for Object Color Perception. Journal of The Franklin Institute 310, 1-26 (1980)

15. McCann, J.J., Hall, J.A., Land, E.H.: Color mondrian experiments: the study of average spectral distributions. Journal of Optical Society America 67, 1380 (1977)

16. Finlayson, G.D., Trezzi, E.: Shades of gray and colour constancy. In: Color Imaging Conference, pp. 37-41 (2004)

17. van de Weijer, J., Gevers, T., Gijsenij, A.: Edge-Based Color Constancy. IEEE Transactions on Image Processing 16(9), 2207-2214 (2007)

18. Kruse, F.A., Lefkoff, A.B., Boardman, J.B., Heidebrecht, K.B., Shapiro, A.T., Barloon, P.J., Goetz, A.F.H.: The Spectral Image Processing System (SIPS) - Interactive Visualization and Analysis of Imaging Spectrometer Data. Remote Sensing of Environment, Special issue on AVIRIS 44(2), 145-163 (1993) 